Volume 9, No.1, January - February 2020

International Journal of Advanced Trends in Computer Science and Engineering

Available Online at http://www.warse.org/IJATCSE/static/pdf/file/ijatcse104912020.pdf

https://doi.org/10.30534/ijatcse/2020/104912020

\title{
Trend Analysis of Hospitals Efficiency Using an Operational Research Technique Approach
}

\author{
Teg Alam ${ }^{1}$, Ayman Mahgoub ${ }^{2}$ \\ ${ }^{1,2}$ College of Business Administration, Prince Sattam Bin Abdulaziz University, Al Kharj, \\ Kingdom of Saudi Arabia \\ t.alam@psau.edu.sa
}

\begin{abstract}
This research focuses on trend analysis of the efficiency of hospitals in twenty regions of the Kingdom of Saudi Arabia. Facilities for health care considered a key component of successful and sustainable community work. Research work in this area is limited in the Saudi context. DEA-an Operational Research approach, well-established methods used to estimate the performance of hospitals.

In this research, the researcher aims to demonstrate the use of "Data Envelopment Analysis" (DEA) to evaluate hospital performance through twenty regions of the Kingdom of Saudi Arabia. The "Basic Radial Models with Variable Returns to scale (VRS) input-oriented" are used to estimate the efficiency of Hospitals.

The DEA method helps administrators recognize inefficient interventions and take the steps required to change them. The input variables used in this analysis include the number of doctors, number of nurses, number of pharmacists, number of medical assistants, number of beds, number of hospitals and number of health centers. The output variables used in this analysis include the number of radiation tests, number of laboratory tests, number of patients (emergency), number of visits (outpatient clinics) and number of patients (number of inpatients at MOH's hospitals). The data obtained from the Ministry of Health's Portal. The lack of productivity should be resolved by increasing budget allocation and only in certain situations by improving policies on resource management.
\end{abstract}

Key words: Efficiency, Trend Analysis, Development, Hospital, Kingdom of Saudi Arabia.

\section{INTRODUCTION}

In the Kingdom of Saudi Arabia, healthcare remains a top priority for the government and this high-potential business sector provides enormous opportunities for growth. In line with the government's Vision 2030 and the National Transformation Plan, the Ministry of Health is expected to spend around US\$ 71 billion over five years ending in 2020.
To improve efficiencies and reduce costs, the KSA government has been actively exploring the role of the private sector in the growth of the Kingdom's healthcare infrastructure.

In the meantime, technology remains a significant factor in the transformation of the KSA healthcare sector in the coming years, with information technology playing a crucial role in providing cost, efficiency, access and resource-related solutions. In 2019, the allocation of the sector's budget increased by 8 per cent to reach SAR172 billion, compared to SAR159 billion for 2018.

Data Envelopment Analysis (DEA) is an operations research approach used in the present study to measure hospital efficiency in twenty regions of the Kingdom of Saudi Arabia.

El-Seoud, M.S.A. (2013) measured the quality of Public Hospitals in Saudi Arabia. Fitzgerald, G. Al Malki, M. and Clark, M. (2011) reported that the efficiency of Saudi Arabia's health care system had improved. Djerdjouri, M. (2013) lays down a framework for measuring hospital performance.

Asma M. A. Bahurmoz (1998) demonstrated that the DEA is a powerful tool for measuring the performance of the primary health care centers in Saudi Arabia. Al Shayea, M.A. (2011) considered deliberation on hospital unit performance. Ibrahim Saeed Aqel and Khaled Atallah Al-Tarawneh (2013) established a connection between health-care quality and patient satisfaction.

Lee, K., Yang, S. and Choi, M. (2009) found that in the United States, non-profitable hospitals were more effective than profitable hospitals. Alam Teg (2018) used DEA to estimate the efficiency scores for hospitals in the Kingdom of Saudi Arabia. Muna Elsdaig and Dua' A. Nassar (2019) studied that Delone and Maclean were used to measuring the quality of success for the KSA Healthcare Information System. Intisar Shadeed Al-Mejibli and Nawaf Rasheed Alharbe (2019) identified the safety risk factors that affect both the usability and web application protection of a web application for healthcare. 


\section{METHODS AND MATERIALS}

\subsection{Mathematical Model}

In this study, there are 20 DMUs, where, 7 inputs and 5 outputs. The efficiency of ' $D M U_{r}$ ' is done by solving the following model (Charnes, A., et al., 1978).

$$
\begin{aligned}
& \max w_{r}=\frac{\sum_{k=1}^{S} v_{k} y_{h r}}{\sum_{j=\Perp}^{7} U_{j} x_{j r}} \\
& \text { s.t. } \quad \frac{\sum_{k=1}^{5} w_{k} y_{k i}}{\sum_{j=1}^{7} u_{j} x_{j i}} \leq 1 ; \forall i_{i} \\
& i=1,2,3, \ldots \ldots, 20 \\
& \text { and } \quad v_{k}, u_{j} \geq 0 ; \forall \mathrm{k}_{j} \mathrm{j} \\
& y_{k i}=\text { quantity of output ' } k \text { ' formed by } \mathrm{DMU}_{1} \\
& x_{\overline{j i}}=\text { quantity of input } \mathrm{j} \text { consumed by } \mathrm{DMU}_{\mathrm{i}} \\
& v_{k}=\text { weight for output } \mathrm{k} \text {, } \\
& u_{j}=\text { weight for input } \mathrm{j} \text {. }
\end{aligned}
$$

Where;

The above nonlinear programming problem converted into a linear program problem (Charnes, A., et al., 1978), as given below.

$$
\begin{gathered}
\max w_{r}=\sum_{k=1}^{5} v_{k} y_{k r} \\
\text { s.t. } \quad \sum_{j=1}^{7} u_{j} x_{j r}=1 \\
\sum_{k=1}^{5} v_{k} y_{k i}-\sum_{j=1}^{7} u_{j} x_{j i} \leq 0 ; \forall i
\end{gathered}
$$$$
\text { and } v_{k}, u_{j} \geq 0 ; \forall \mathrm{k}_{e} \mathrm{j}
$$

If $w_{r}$ is one, then $D M U_{r}$ is efficient relative to other units. If, $w_{r}$ is less than one, then the $D M U_{r}$ is inefficient. Later, the BCC model (Banker, R.D., et al., 1984) modified the original CCR linear programming by adding a convexity constraint.

\subsection{Data}

\begin{tabular}{|c|c|}
\hline DMUs & Region \\
\hline $\mathbf{D M U}_{1}$ & Riyadh Health Region \\
\hline $\mathrm{DMU}_{2}$ & The Holy Capital Region \\
\hline $\mathrm{DMU}_{3}$ & Directorate of Health Affairs in Jeddah \\
\hline $\mathrm{DMU}_{4}$ & Directorate of Health Affairs in Taif \\
\hline $\mathrm{DMU}_{5}$ & Directorate of Health Affairs in Ahsa \\
\hline $\mathrm{DMU}_{6}$ & Directorate of Health Affairs in Hafr Al-Batin \\
\hline $\mathbf{D M U}_{7}$ & Health Affairs in Sharqia \\
\hline $\mathrm{DMU}_{8}$ & Tabuk Region - Directorate General of Health Affairs \\
\hline DMU $_{9}$ & Director of Health Affairs in Qurayat Region \\
\hline $\mathrm{DMU}_{10}$ & Northern Front Health Affairs \\
\hline $\mathrm{DMU}_{11}$ & $\begin{array}{l}\text { Al-Jouf Region - Directorate General of Health } \\
\text { Affairs }\end{array}$ \\
\hline $\mathrm{DMU}_{12}$ & $\begin{array}{l}\text { Madina Al Munawwarah Region - General } \\
\text { Directorate of Health Affairs }\end{array}$ \\
\hline $\mathrm{DMU}_{13}$ & $\begin{array}{l}\text { Qassim Region - Directorate General for Health } \\
\text { Affairs }\end{array}$ \\
\hline $\mathrm{DMU}_{14}$ & Hail Region - Directorate General of Health Affairs \\
\hline $\mathrm{DMU}_{15}$ & Assir Region - Directorate General of Health Affairs \\
\hline $\mathrm{DMU}_{16}$ & Matafa Jizan - Directorate General of Health Affairs \\
\hline $\mathrm{DMU}_{17}$ & Najran Region - Directorate General of Health Affairs \\
\hline $\mathrm{DMU}_{18}$ & Directorate of Health Affairs Bisha \\
\hline $\mathrm{DMU}_{19}$ & Al Baha Area - General Directorate of Health Affairs \\
\hline $\mathbf{D M U}_{20}$ & Directorate of Health Affairs in Qanafah \\
\hline
\end{tabular}

The data used in this analysis include hospitals in twenty regions of the Kingdom of Saudi Arabia. The dataset (1435 Hijri-1439 Hijri) for this analysis is collected from the Ministry of Health $(\mathrm{MOH})$ database. This research utilized DEA Online software to measure hospital efficiency scores through the Variable return to the scale of the Basic Radial (input-oriented) model.

\subsection{Decision-Making Units}

The decision-making units are defined in the following table 1 .

Table 1: Decision- making units of hospitals region-wise 


\section{RESULTS AND DISCUSSION}

Table 2: Trend Analysis of Hospitals (Efficiencies of DMUs)

\begin{tabular}{crrrrr}
\hline DMUs & $\mathbf{1 4 3 5}$ & $\mathbf{1 4 3 6}$ & $\mathbf{1 4 3 7}$ & $\mathbf{1 4 3 8}$ & $\mathbf{1 4 3 9}$ \\
\hline $\mathbf{D M U}_{\mathbf{1}}$ & 1 & 1 & 1 & 1 & 1 \\
\hline $\mathbf{D M U}_{\mathbf{2}}$ & 1 & 1 & 1 & 1 & 1 \\
$\mathbf{D M U}_{\mathbf{3}}$ & 1 & 1 & 0.997 & 1 & 0.961 \\
\hline $\mathbf{D M U}_{\mathbf{4}}$ & 1 & 1 & 0.862 & 0.925 & 0.845 \\
\hline $\mathbf{D M U}_{\mathbf{5}}$ & 1 & 1 & 1 & 1 & 1 \\
\hline $\mathbf{D M U}_{\mathbf{6}}$ & 1 & 1 & 1 & 1 & 1 \\
\hline $\mathbf{D M U}_{\mathbf{7}}$ & 1 & 1 & 1 & 1 & 1 \\
\hline $\mathbf{D M U}_{\mathbf{8}}$ & 1 & 1 & 0.908 & 0.936 & 0.704 \\
\hline $\mathbf{D M U}_{\mathbf{9}}$ & 1 & 1 & 1 & 1 & 1 \\
\hline $\mathbf{D M U}_{\mathbf{1 0}}$ & 1 & 1 & 0.834 & 1 & 1 \\
\hline $\mathbf{D M U}_{\mathbf{1 1}}$ & 0.9119 & 0.807 & 1 & 1 & 1 \\
\hline $\mathbf{D M U}_{\mathbf{1 2}}$ & 1 & 1 & 1 & 1 & 1 \\
\hline $\mathbf{D M U}_{\mathbf{1 3}}$ & 1 & 1 & 1 & 1 & 1 \\
\hline $\mathbf{D M U}_{\mathbf{1 4}}$ & 1 & 1 & 1 & 1 & 0.985 \\
\hline $\mathbf{D M U}_{\mathbf{1 5}}$ & 1 & 1 & 1 & 1 & 1 \\
\hline $\mathbf{D M U}_{\mathbf{1 6}}$ & 1 & 1 & 1 & 1 & 1 \\
\hline $\mathbf{D M U}_{\mathbf{1 7}}$ & 0.858 & 0.8617 & 1 & 0.926 & 1 \\
\hline $\mathbf{D M U}_{\mathbf{1 8}}$ & 1 & 1 & 1 & 1 & 1 \\
\hline $\mathbf{D M U}_{\mathbf{1 9}}$ & 1 & 1 & 1 & 1 & 1 \\
\hline $\mathbf{D M U}_{\mathbf{2 0}}$ & 1 & 1 & 1 & 1 & 1 \\
\hline & & & & & \\
\hline & 1 & 1 & 1 & 1 \\
\hline
\end{tabular}

\section{Trend Analysis of Hospitals}

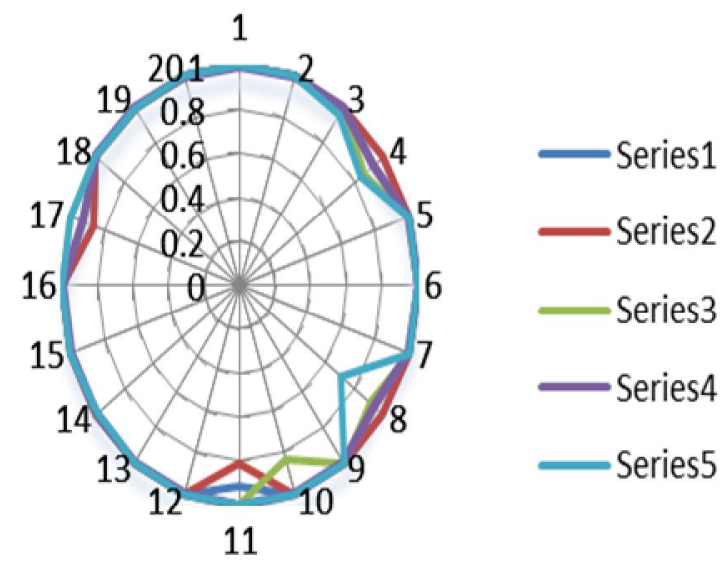

Figure 1: Graphical presentation of trend analysis of hospitals

From the table2, we revealed that the Hospitals are successful in twenty regions of the Kingdom of Saudi Arabia. If the efficiency score is less than one, the DMU is inefficient; such hospitals should use their resources effectively to produce outputs. In the year 1435, DMU11 and DMU17; in year 1436 DMU11 and DMU17; in year 1437, DMU3, DMU4, DMU8 and DMU10; in year 1438 DMU4, DMU8 and DMU17; in year 1439 DMU3, DMU4, DMU8 and DMU14 are closer to 1, showing that such hospitals should improve their performance and become more effective soon. The study shows that nearly all hospitals in twenty regions of the Kingdom of Saudi Arabia used their services efficiently. We have also seen from table 1 that DMU 3, DMU 4, DMU 8 and DMU17 need to be given more emphasis.

The findings of the study are useful and important for hospital administrators and policymakers who can help them make decisions and control their resources.

\subsection{Input and Output Slack Variables}

If the efficiency of a DMU is equal to one, and there are zero slack variables, then this is the efficiency of Pareto. If the efficiency of a DMU is equal to one, and there are non-zero slack variables, then it is known as the low efficiency. Input slack and output slack variables relating to each DMU are given respectively in tables 2,3,4,5,6,7,8,9,10 and 11 .

Table 3: Input slack variables 1435

\begin{tabular}{|c|c|c|c|c|c|c|c|}
\hline DMUs & Input-1 & Input-2 & Input-3 & Input-4 & Input-5 & Input-6 & Input-7 \\
\hline $\mathrm{DMU}_{1}$ & 0 & 0 & 0 & 0 & 0 & 0 & 0 \\
\hline $\mathrm{DMU}_{2}$ & 0 & 0 & 0 & 0 & 0 & 0 & 0 \\
\hline $\mathrm{DMU}_{3}$ & 0 & 0 & 0 & 0 & 0 & 0 & 0 \\
\hline $\mathrm{DMU}_{4}$ & 0 & 0 & 0 & 0 & 0 & 0 & 0 \\
\hline $\mathrm{DMU}_{5}$ & 0 & 0 & 0 & 0 & 0 & 0 & 0 \\
\hline $\mathrm{DMU}_{6}$ & 0 & 0 & 0 & 0 & 0 & 0 & 0 \\
\hline $\mathrm{DMU}_{7}$ & 0 & 0 & 0 & 0 & 0 & 0 & 0 \\
\hline $\mathrm{DMU}_{8}$ & 0 & 0 & 0 & 0 & 0 & 0 & 0 \\
\hline $\mathrm{DMU}_{9}$ & 0 & 0 & 0 & 0 & 0 & 0 & 0 \\
\hline $\mathrm{DMU}_{10}$ & 0 & 0 & 0 & 0 & 0 & 0 & 0 \\
\hline $\mathrm{DMU}_{11}$ & 92.465 & 478.932 & 0 & 150.266 & 335.736 & 0 & 0 \\
\hline $\mathrm{DMU}_{12}$ & 0 & 0 & 0 & 0 & 0 & 0 & 0 \\
\hline $\operatorname{DMU}_{13}$ & 0 & 0 & 0 & 0 & 0 & 0 & 0 \\
\hline $\mathrm{DMU}_{14}$ & 0 & 0 & 0 & 0 & 0 & 0 & 0 \\
\hline $\mathrm{DMU}_{15}$ & 0 & 0 & 0 & 0 & 0 & 0 & 0 \\
\hline DMU $_{16}$ & 0 & 0 & 0 & 0 & 0 & 0 & 0 \\
\hline $\mathbf{D M U}_{17}$ & 83.745 & 166.322 & 16.432 & 346.231 & 0 & 0 & 0 \\
\hline $\mathrm{DMU}_{18}$ & 0 & 0 & 0 & 0 & 0 & 0 & 0 \\
\hline $\mathrm{DMU}_{19}$ & 0 & 0 & 0 & 0 & 0 & 0 & 0 \\
\hline $\mathrm{DMU}_{20}$ & 0 & 0 & 0 & 0 & 0 & 0 & 0 \\
\hline
\end{tabular}

Table 3 shows that to achieve the desired performance, DMU11 required slack variables for input 1, input 2, input 4 and input 5 , respectively $92.465,478.932,150.266$ and 335.736. To obtain the desired efficiency, DMU17 required slack variables for Input 1 Input2, Input3 and Input4 are $83.745,166.322,16.432$, and 346.231 , respectively. 
Table 4: Output slack variables 1435

\begin{tabular}{lrrrrr}
\hline DMUS & Output-1 & Output-2 & Output-3 & Output-4 & Output-5 \\
\hline $\mathbf{D M U}_{1}$ & 0 & 0 & 0 & 0 & 0 \\
\hline $\mathbf{D M U}_{2}$ & 0 & 0 & 0 & 0 & 0 \\
\hline $\mathbf{D M U}_{3}$ & 0 & 0 & 0 & 0 & 0 \\
\hline $\mathbf{D M U}_{4}$ & 0 & 0 & 0 & 0 & 0 \\
\hline $\mathbf{D M U}_{5}$ & 0 & 0 & 0 & 0 & 0 \\
\hline $\mathbf{D M U}_{6}$ & 0 & 0 & 0 & 0 & 0 \\
\hline $\mathbf{D M U}_{7}$ & 0 & 0 & 0 & 0 & 0 \\
\hline $\mathbf{D M U}_{\mathbf{8}}$ & 0 & 0 & 0 & 0 & 0 \\
\hline $\mathbf{D M U}_{9}$ & 0 & 0 & 0 & 0 & 0 \\
\hline $\mathbf{D M U}_{10}$ & 0 & 0 & 0 & 0 & 0 \\
\hline $\mathbf{D M U}_{11}$ & 15823.41 & 507365.7 & 0 & 0 & 5309.622 \\
\hline $\mathbf{D M U}_{12}$ & 0 & 0 & 0 & 0 & 0 \\
\hline $\mathbf{D M U}_{13}$ & 0 & 0 & 0 & 0 & 0 \\
\hline $\mathbf{D M U}_{14}$ & 0 & 0 & 0 & 0 & 0 \\
\hline $\mathbf{D M U}_{15}$ & 0 & 0 & 0 & 0 & 0 \\
\hline $\mathbf{D M U}_{16}$ & 0 & 0 & 0 & 0 & 0 \\
\hline $\mathbf{D M U}_{17}$ & 0 & 921739.7 & 40989.96 & 0 & 0 \\
\hline $\mathbf{D M U}_{18}$ & 0 & 0 & 0 & 0 & 0 \\
\hline $\mathbf{D M U}_{19}$ & 0 & 0 & 0 & 0 & 0 \\
\hline $\mathbf{D M U}_{\mathbf{2 0}}$ & 0 & 0 & 0 & 0 & 0 \\
\hline $\mathbf{D n}_{\mathbf{1}}$ & 0 & 0 & 0 & 0 \\
\hline
\end{tabular}

In order to achieve the target performance, it is evident from Table 4, DMU 11 required slack variables for output 1 , output 2 and output 5 are 15823.41, 507365.7 and 5309.622. DMU 17 needed slack variables for output 2 and output 3 respectively are 921739.7 and 40989.96, in order to achieve the target efficiency.

Table 5: Input slack variables 1436

\begin{tabular}{lrrrrrrr}
\hline DMUs & Input-1 & Input-2 & Input-3 & Input-4 & Input-5 & Input-6 & Input-7 \\
\hline $\mathbf{D M U}_{\mathbf{1}}$ & 0 & 0 & 0 & 0 & 0 & 0 & 0 \\
\hline $\mathbf{D M U}_{2}$ & 0 & 0 & 0 & 0 & 0 & 0 & 0 \\
\hline $\mathbf{D M U}_{\mathbf{3}}$ & 0 & 0 & 0 & 0 & 0 & 0 & 0 \\
\hline $\mathbf{D M U}_{4}$ & 0 & 0 & 0 & 0 & 0 & 0 & 0 \\
\hline $\mathbf{D M U}_{\mathbf{5}}$ & 0 & 0 & 0 & 0 & 0 & 0 & 0 \\
\hline $\mathbf{D M U}_{\mathbf{6}}$ & 0 & 0 & 0 & 0 & 0 & 0 & 0 \\
\hline $\mathbf{D M U}_{7}$ & 0 & 0 & 0 & 0 & 0 & 0 & 0 \\
\hline $\mathbf{D M U}_{\mathbf{8}}$ & 0 & 0 & 0 & 0 & 0 & 0 & 0 \\
\hline $\mathbf{D M U}_{\mathbf{9}}$ & 0 & 0 & 0 & 0 & 0 & 0 & 0 \\
\hline $\mathbf{D M U}_{\mathbf{1 0}}$ & 0 & 0 & 0 & 0 & 0 & 0 & 0 \\
\hline $\mathbf{D M U}_{11}$ & 49.205 & 466.853 & 0 & 6.005 & 244.991 & 0.567 & 0 \\
\hline $\mathbf{D M U}_{12}$ & 0 & 0 & 0 & 0 & 0 & 0 & 0 \\
\hline $\mathbf{D M U}_{13}$ & 0 & 0 & 0 & 0 & 0 & 0 & 0 \\
\hline $\mathbf{D M U}_{14}$ & 0 & 0 & 0 & 0 & 0 & 0 & 0 \\
\hline $\mathbf{D M U}_{15}$ & 0 & 0 & 0 & 0 & 0 & 0 & 0 \\
\hline $\mathbf{D M U}_{16}$ & 0 & 0 & 0 & 0 & 0 & 0 & 0 \\
\hline $\mathbf{D M U}_{17}$ & 138.39 & 357.754 & 30.772 & 494.968 & 0 & 1.152 & 0 \\
\hline $\mathbf{D M U}_{18}$ & 0 & 0 & 0 & 0 & 0 & 0 & 0 \\
\hline $\mathbf{D M U}_{19}$ & 0 & 0 & 0 & 0 & 0 & 0 & 0 \\
\hline $\mathbf{D M U}_{20}$ & 0 & 0 & 0 & 0 & 0 & 0 & 0 \\
\hline & & & & & & & \\
\hline
\end{tabular}

It is observed that from table 5, DMU11 needed slack variables for input 1, input 2, input 4,input 5 and input 6 to achieve the desirable efficiency, respectively 49.205, 466.853,6.005, 244.991and 0.567. To achieve the desired efficiency, DMU17 required slack variables for Input1 Input2, Input3, Input4 and input 6 are 138.39, 357.754, 30.772, 494.968 and1.152 respectively.

Table 6: Output slack variables 1436

\begin{tabular}{|c|c|c|c|c|c|}
\hline DMUS & Output-1 & Output-2 & Output-3 & Output-4 & Output-5 \\
\hline DMU $_{1}$ & 0 & 0 & ( & $\overline{0}$ & 0 \\
\hline $\mathbf{D M U}_{2}$ & 0 & 0 & ( & 0 & 0 \\
\hline $\mathrm{DMU}_{3}$ & 0 & 0 & ( & 0 & 0 \\
\hline $\mathbf{D M U}_{4}$ & 0 & 0 & ( & 0 & 0 \\
\hline DMU $_{5}$ & 0 & 0 & ( & 0 & 0 \\
\hline $\mathrm{DMU}_{6}$ & 0 & 0 & c & $c$ & 0 \\
\hline $\mathbf{D M U}_{7}$ & 0 & 0 & ( & c & 0 \\
\hline DMU $_{8}$ & 0 & 0 & ( & 0 & 0 \\
\hline DMU, $_{9}$ & 0 & 0 & ( & c & 0 \\
\hline $\mathrm{DMU}_{10}$ & 0 & 0 & ( & 0 & 0 \\
\hline $\mathbf{D M U}_{11}$ & 12765.52 & 621352.5 & ( & c & 494.954 \\
\hline $\mathrm{DMU}_{12}$ & 0 & 0 & ( & c & 0 \\
\hline $\mathrm{DMU}_{13}$ & 0 & 0 & ( & c & 0 \\
\hline DMU $_{14}$ & 0 & 0 & ( & $c$ & 0 \\
\hline DMU $_{15}$ & 0 & 0 & ( & c & 0 \\
\hline $\mathrm{DMU}_{16}$ & 0 & 0 & ( & c & 0 \\
\hline $\mathbf{D M U}_{17}$ & 9761.421 & 785740.3 & ( & c & 0 \\
\hline $\mathrm{DMU}_{18}$ & 0 & 0 & ( & c & 0 \\
\hline DMU $_{19}$ & 0 & 0 & ( & c & 0 \\
\hline $\mathbf{D M U}_{20}$ & 0 & 0 & ( & c & 0 \\
\hline
\end{tabular}

It is evident from Table 6, in order to achieve the target performance, DMU 11 required slack variables for output 1 , output 2 and output 5 are 12765.52, 621352.5 and 494.954. In order to achieve the target performance, DMU 17 required slack variables for output 1 and output 1 are 9761.421 and 785740.3 respectively.

Table 7: Input slack variables 1437

\begin{tabular}{crrrrrrr}
\hline DMUs & Input-1 & Input-2 & Input-3 & Input-4 & Input-5 & Input-6 & Input-7 \\
\hline DMU $_{\mathbf{1}}$ & 0 & 0 & 0 & 0 & 0 & 0 & 0 \\
$\mathbf{D M U}_{\mathbf{2}}$ & 0 & 0 & 0 & 0 & 0 & 0 & 0 \\
$\mathbf{D M U}_{\mathbf{3}}$ & 325.7 & 940.0 & 0 & 1047.2 & 668.6 & 1.6 & 0 \\
$\mathbf{D M U}_{\mathbf{4}}$ & 0 & 623.5 & 7.3 & 0 & 379.3 & 0 & 8.72 \\
$\mathbf{D M U}_{\mathbf{5}}$ & 0 & 0 & 0 & 0 & 0 & 0 & 0 \\
$\mathbf{D M U}_{\mathbf{6}}$ & 0 & 0 & 0 & 0 & 0 & 0 & 0 \\
$\mathbf{D M U}_{\mathbf{7}}$ & 0 & 0 & 0 & 0 & 0 & 0 & 0 \\
$\mathbf{D M U}_{\mathbf{8}}$ & 36.52 & 204.7 & 8.9 & 0 & 186.8 & 0.2 & 0 \\
$\mathbf{D M U}_{\mathbf{9}}$ & 0 & 0 & 0 & 0 & 0 & 0 & 0 \\
$\mathbf{D M U}_{\mathbf{1 0}}$ & 151.6 & 436.5 & 9.3 & 0 & 335 & 1.5 & 0 \\
$\mathbf{D M U}_{\mathbf{1 1}}$ & 0 & 0 & 0 & 0 & 0 & 0 & 0 \\
$\mathbf{D M U}_{\mathbf{1 2}}$ & 0 & 0 & 0 & 0 & 0 & 0 & 0 \\
$\mathbf{D M U}_{\mathbf{1 3}}$ & 0 & 0 & 0 & 0 & 0 & 0 & 0 \\
$\mathbf{D M U}_{\mathbf{1 4}}$ & 0 & 0 & 0 & 0 & 0 & 0 & 0 \\
$\mathbf{D M U}_{\mathbf{1 5}}$ & 0 & 0 & 0 & 0 & 0 & 0 & 0 \\
$\mathbf{D M U}_{\mathbf{1 6}}$ & 0 & 0 & 0 & 0 & 0 & 0 & 0 \\
$\mathbf{D M U}_{\mathbf{1 7}}$ & 0 & 0 & 0 & 0 & 0 & 0 & 0 \\
$\mathbf{D M U}_{\mathbf{1 8}}$ & 0 & 0 & 0 & 0 & 0 & 0 & 0 \\
$\mathbf{D M U}_{\mathbf{1 9}}$ & 0 & 0 & 0 & 0 & 0 & 0 & 0 \\
$\mathbf{D M U}_{\mathbf{2 0}}$ & 0 & 0 & 0 & 0 & 0 & 0 & 0 \\
\hline & & & & & & &
\end{tabular}


Table 7 shows that DMU3 needed slack variables for input 1 , input 2, input 4, input 5 and input 6; DMU4 required slacks for input 2, input 3, input 5 and input 7; DMU8 required slack variables for input 1 , input 2 , input 3 , input 5 and input 6 ; and DMU10 required slack variables for input 1 , input 2 , input 3 , input 5 and input 6 ; to achieve the desired output.

Table 8: Output slack variables 1437

\begin{tabular}{crrrrr}
\hline DMUS & Output-1 & Output-2 & Output-3 & Output-4 & Output-5 \\
\hline $\mathbf{D M U}_{\mathbf{1}}$ & 0 & 0 & 0 & 0 & 0 \\
\hline $\mathbf{D M U}_{\mathbf{2}}$ & 0 & 0 & 0 & 0 & 0 \\
\hline $\mathbf{D M U}_{\mathbf{3}}$ & 0 & 1770755 & 304978.3 & 0 & 4394.758 \\
\hline $\mathbf{D M U}_{\mathbf{4}}$ & 152029.4 & 3557911 & 0 & 0 & 0 \\
\hline $\mathbf{D M U}_{\mathbf{5}}$ & 0 & 0 & 0 & 0 & 0 \\
\hline $\mathbf{D M U}_{\mathbf{6}}$ & 0 & 0 & 0 & 0 & 0 \\
\hline $\mathbf{D M U}_{7}$ & 0 & 0 & 0 & 0 & 0 \\
\hline $\mathbf{D M U}_{\mathbf{8}}$ & 146426.2 & 2744576 & 0 & 0 & 0 \\
\hline $\mathbf{D M U}_{\mathbf{9}}$ & 0 & 0 & 0 & 0 & 0 \\
\hline $\mathbf{D M U}_{\mathbf{1 0}}$ & 3037.02 & 1549340 & 0 & 0 & 0 \\
\hline $\mathbf{D M U}_{\mathbf{1 1}}$ & 0 & 0 & 0 & 0 & 0 \\
\hline $\mathbf{D M U}_{\mathbf{1 2}}$ & 0 & 0 & 0 & 0 & 0 \\
\hline $\mathbf{D M U}_{\mathbf{1 3}}$ & 0 & 0 & 0 & 0 & 0 \\
\hline $\mathbf{D M U}_{\mathbf{1 4}}$ & 0 & 0 & 0 & 0 & 0 \\
\hline $\mathbf{D M U}_{\mathbf{1 5}}$ & 0 & 0 & 0 & 0 & 0 \\
\hline $\mathbf{D M U}_{\mathbf{1 6}}$ & 0 & 0 & 0 & 0 & 0 \\
\hline $\mathbf{D M U}_{\mathbf{1 7}}$ & 0 & 0 & 0 & 0 & 0 \\
\hline $\mathbf{D M U}_{\mathbf{1 8}}$ & 0 & 0 & 0 & 0 & 0 \\
\hline $\mathbf{D M U}_{\mathbf{1 9}}$ & 0 & 0 & 0 & 0 & 0 \\
\hline $\mathbf{D M U}_{\mathbf{2 0}}$ & 0 & 0 & 0 & 0 & 0 \\
\hline $\mathbf{T a b}_{\mathbf{1}}$ & 0 & 0 & 0 & 0 \\
\hline
\end{tabular}

Table 8 shows that to achieve the target performance ;DMU 3 needed slack variables for output 2, output 3 and output 5; DMU 4 required slacks for output 1and output 2; DMU 8 required slack variables for output 1 and output 2 ; DMU 10 required slack for output 1 , and output 2 .

Table 9: Input slack variables 1438

\begin{tabular}{lrrrrrrr}
\hline DMUs & Input-1 & Input-2 & Input-3 & Input-4 & Input-5 & Input-6 & Input-7 \\
\hline $\mathbf{D M U}_{\mathbf{1}}$ & 0 & 0 & 0 & 0 & 0 & 0 & 0 \\
$\mathbf{D M U}_{\mathbf{2}}$ & 0 & 0 & 0 & 0 & 0 & 0 & 0 \\
$\mathbf{D M U}_{\mathbf{3}}$ & 0 & 0 & 0 & 0 & 0 & 0 & 0 \\
$\mathbf{D M U}_{\mathbf{4}}$ & 23.1 & 442.7 & 16.7 & 0 & 545.5 & 0.9 & 0 \\
$\mathbf{D M U}_{\mathbf{5}}$ & 0 & 0 & 0 & 0 & 0 & 0 & 0 \\
$\mathbf{D M U}_{\mathbf{6}}$ & 0 & 0 & 0 & 0 & 0 & 0 & 0 \\
$\mathbf{D M U}_{\mathbf{7}}$ & 0 & 0 & 0 & 0 & 0 & 0 & 0 \\
$\mathbf{D M U}_{\mathbf{8}}$ & 67.9 & 276.9 & 7.2 & 0 & 0 & 0 & 0 \\
$\mathbf{D M U}_{\mathbf{9}}$ & 0 & 0 & 0 & 0 & 0 & 0 & 0 \\
$\mathbf{D M U}_{\mathbf{1 0}}$ & 0 & 0 & 0 & 0 & 0 & 0 & 0 \\
$\mathbf{D M U}_{\mathbf{1 1}}$ & 0 & 0 & 0 & 0 & 0 & 0 & 0 \\
$\mathbf{D M U}_{\mathbf{1 2}}$ & 0 & 0 & 0 & 0 & 0 & 0 & 0 \\
$\mathbf{D M U}_{\mathbf{1 3}}$ & 0 & 0 & 0 & 0 & 0 & 0 & 0 \\
$\mathbf{D M U}_{\mathbf{1 4}}$ & 0 & 0 & 0 & 0 & 0 & 0 & 0 \\
$\mathbf{D M U}_{\mathbf{1 5}}$ & 0 & 0 & 0 & 0 & 0 & 0 & 0 \\
$\mathbf{D M U}_{\mathbf{1 6}}$ & 0 & 0 & 0 & 0 & 0 & 0 & 0 \\
$\mathbf{D M U}_{\mathbf{1 7}}$ & 84.8 & 0 & 48.2 & 444.3 & 0 & 1.3 & 0 \\
$\mathbf{D M U}_{\mathbf{1 8}}$ & 0 & 0 & 0 & 0 & 0 & 0 & 0 \\
$\mathbf{D M U}_{\mathbf{1 9}}$ & 0 & 0 & 0 & 0 & 0 & 0 & 0 \\
$\mathbf{D M U}_{\mathbf{2 0}}$ & 0 & 0 & 0 & 0 & 0 & 0 & 0 \\
\hline${ }^{-8}$ & & & & & & & \\
\hline
\end{tabular}

It is seen that from table 9, DMU4 required slack variables for input 1 , input 2 , input 3 , input 5 and input 6; DMU8 needed slack for input 1, input 2 and input 3 and DMU17 needed slack variables for input 1 , input 3 , input 4 and input 6 ; to accomplish the ideal effectiveness.

Table 10: Output slack variables 1438

\begin{tabular}{lrrrrr}
\hline DMUS & Output-1 & Output-2 & Output-3 & Output-4 & Output-5 \\
\hline $\mathbf{D M U}_{1}$ & 0 & 0 & 0 & 0 & 0 \\
\hline $\mathbf{D M U}_{2}$ & 0 & 0 & 0 & 0 & 0 \\
\hline $\mathbf{D M U}_{3}$ & 0 & 0 & 0 & 0 & 0 \\
\hline $\mathbf{D M U}_{4}$ & 124375.8 & 1114127 & 0 & 0 & 0 \\
\hline $\mathbf{D M U}_{5}$ & 0 & 0 & 0 & 0 & 0 \\
\hline $\mathbf{D M U}_{6}$ & 0 & 0 & 0 & 0 & 0 \\
\hline $\mathbf{D M U}_{7}$ & 0 & 0 & 0 & 0 & 0 \\
\hline $\mathbf{D M U}_{\mathbf{8}}$ & 58063.65 & 2385039 & 0 & 0 & 7547.522 \\
\hline $\mathbf{D M U}_{9}$ & 0 & 0 & 0 & 0 & 0 \\
\hline $\mathbf{D M U}_{10}$ & 0 & 0 & 0 & 0 & 0 \\
\hline $\mathbf{D M U}_{11}$ & 0 & 0 & 0 & 0 & 0 \\
\hline $\mathbf{D M U}_{12}$ & 0 & 0 & 0 & 0 & 0 \\
\hline $\mathbf{D M U}_{13}$ & 0 & 0 & 0 & 0 & 0 \\
\hline $\mathbf{D M U}_{14}$ & 0 & 0 & 0 & 0 & 0 \\
\hline $\mathbf{D M U}_{15}$ & 0 & 0 & 0 & 0 & 0 \\
\hline $\mathbf{D M U}_{16}$ & 0 & 0 & 0 & 0 & 0 \\
\hline $\mathbf{D M U}_{17}$ & 8443.505 & 2574656 & 0 & 76046.86 & 0 \\
\hline $\mathbf{D M U}_{18}$ & 0 & 0 & 0 & 0 & 0 \\
\hline $\mathbf{D M U}_{19}$ & 0 & 0 & 0 & 0 & 0 \\
\hline $\mathbf{D M U}_{20}$ & 0 & 0 & 0 & 0 & 0 \\
\hline $\mathbf{T}_{21}$ & 0 & 0 & 0 & 0 \\
\hline
\end{tabular}

Table 10 shows that to achieve the target performance; DMU 4 required slack variables at output 1and output 2; DMU 8 needed slack variables at output 1 , output 2 and output 5; DMU 17 required slack variables at output 1 , output 2 and output 5 .

Table 11:. Input slack variables 1439

\begin{tabular}{lrrrrrrr}
\hline DMUs & Input-1 & Input-2 & Input-3 & Input-4 & Input-5 & Input-6 & Input-7 \\
\hline $\mathbf{D M U}_{\mathbf{1}}$ & 0 & 0 & 0 & 0 & 0 & 0 & 0 \\
$\mathbf{D M U}_{\mathbf{2}}$ & 0 & 0 & 0 & 0 & 0 & 0 & 0 \\
$\mathbf{D M U}_{\mathbf{3}}$ & 750.1 & 1077.6 & 0 & 1522.1 & 416.1 & 1.6 & 0 \\
$\mathbf{D M U}_{\mathbf{4}}$ & 120.3 & 442.3 & 13.1 & 0 & 0 & 1.3 & 0 \\
\hline $\mathbf{D M U}_{\mathbf{5}}$ & 0 & 0 & 0 & 0 & 0 & 0 & 0 \\
$\mathbf{D M U}_{\mathbf{6}}$ & 0 & 0 & 0 & 0 & 0 & 0 & 0 \\
\hline $\mathbf{D M U}_{\mathbf{7}}$ & 0 & 0 & 0 & 0 & 0 & 0 & 0 \\
$\mathbf{D M U}_{\mathbf{8}}$ & 64.4 & 0 & 4.6 & 0 & 0 & 0 & 0 \\
\hline $\mathbf{D M U}_{\mathbf{9}}$ & 0 & 0 & 0 & 0 & 0 & 0 & 0 \\
\hline $\mathbf{D M U}_{\mathbf{1 0}}$ & 0 & 0 & 0 & 0 & 0 & 0 & 0 \\
$\mathbf{D M U}_{\mathbf{1 1}}$ & 0 & 0 & 0 & 0 & 0 & 0 & 0 \\
$\mathbf{D M U}_{\mathbf{1 2}}$ & 0 & 0 & 0 & 0 & 0 & 0 & 0 \\
$\mathbf{D M U}_{\mathbf{1 3}}$ & 0 & 0 & 0 & 0 & 0 & 0 & 0 \\
\hline $\mathbf{D M U}_{\mathbf{1 4}}$ & 94.9 & 0 & 0 & 127.9 & 329.8 & 2.9 & 35.1 \\
\hline $\mathbf{D M U}_{\mathbf{1 5}}$ & 0 & 0 & 0 & 0 & 0 & 0 & 0 \\
$\mathbf{D M U}_{\mathbf{1 6}}$ & 0 & 0 & 0 & 0 & 0 & 0 & 0 \\
$\mathbf{D M U}_{\mathbf{1 7}}$ & 0 & 0 & 0 & 0 & 0 & 0 & 0 \\
\hline $\mathbf{D M U}_{\mathbf{1 8}}$ & 0 & 0 & 0 & 0 & 0 & 0 & 0 \\
$\mathbf{D M U}_{\mathbf{1 9}}$ & 0 & 0 & 0 & 0 & 0 & 0 & 0 \\
\hline $\mathbf{D M U}_{\mathbf{2 0}}$ & 0 & 0 & 0 & 0 & 0 & 0 & 0 \\
\hline & & & & & & & \\
\hline
\end{tabular}


It is seen that from table 11; DMU3 required slack variables for input 1 , input 2, input 4 , input 5 and input 6; DMU4 required slack variables for input 1 , input 2 , input 3 and input 6; DMU8 needed slack variables for input 1and input 2 and DMU14 needed slack variables for input 1 , input 4 , input 5 , input 6 and input 7; to accomplish the ideal productivity.

Table12: Output slack variables 1439

\begin{tabular}{lrrrrr}
\hline DMUS & Output-1 & Output-2 & Output-3 & Output-4 & Output-5 \\
\hline DMU $_{1}$ & 0 & 0 & 0 & 0 & 0 \\
\hline $\mathbf{D M U}_{2}$ & 0 & 0 & 0 & 0 & 0 \\
\hline $\mathbf{D M U}_{3}$ & 0 & 564454.7 & 338259.6 & 186323.3 & 11526.7 \\
\hline $\mathbf{D M U}_{4}$ & 44856.18 & 5252664 & 0 & 0 & 0 \\
\hline $\mathbf{D M U}_{5}$ & 0 & 0 & 0 & 0 & 0 \\
\hline $\mathbf{D M U}_{6}$ & 0 & 0 & 0 & 0 & 0 \\
\hline $\mathbf{D M U}_{7}$ & 0 & 0 & 0 & 0 & 0 \\
\hline $\mathbf{D M U}_{8}$ & 0 & 1665235 & 0 & 20437.7 & 0 \\
\hline $\mathbf{D M U}_{9}$ & 0 & 0 & 0 & 0 & 0 \\
\hline $\mathbf{D M U}_{10}$ & 0 & 0 & 0 & 0 & 0 \\
\hline $\mathbf{D M U}_{11}$ & 0 & 0 & 0 & 0 & 0 \\
\hline $\mathbf{D M U}_{12}$ & 0 & 0 & 0 & 0 & 0 \\
\hline $\mathbf{D M U}_{13}$ & 0 & 0 & 0 & 0 & 0 \\
\hline $\mathbf{D M U}_{14}$ & 0 & 1888873 & 0 & 84047.6 & 1564.9 \\
\hline $\mathbf{D M U}_{15}$ & 0 & 0 & 0 & 0 & 0 \\
\hline $\mathbf{D M U}_{16}$ & 0 & 0 & 0 & 0 & 0 \\
\hline $\mathbf{D M U}_{17}$ & 0 & 0 & 0 & 0 & 0 \\
\hline $\mathbf{D M U}_{18}$ & 0 & 0 & 0 & 0 & 0 \\
\hline $\mathbf{D M U}_{19}$ & 0 & 0 & 0 & 0 & 0 \\
\hline $\mathbf{D M U}_{20}$ & 0 & 0 & 0 & 0 & 0 \\
\hline $\mathbf{T a b l e}_{12}$ & & 0 & 0 & 0 & 0 \\
\hline
\end{tabular}

Table 12 shows that to achieve the target performance; DMU 3 required slack variables at output 2, output 3 , output 4 and output 5; DMU 4 required slack variables at output 1and output 2; DMU 8 needed slack variables at output 2 and output 4; DMU 14 required slacks at output 2, output 4 and output 5.

\section{CONCLUSION AND SUGGESTION}

This analysis used the DEA approach to assess the trend of hospital efficiencies in twenty areas of the Kingdom of Saudi Arabia. The outcomes have shown that practically all Hospitals were proficient in terms of Basic Radial Models input-oriented with Variable Returns to scale (VRS).

The results indicated that the hospitals used their resources efficiently to generate all of the specified output. The productivity ratings showed whether or not hospitals need enhancement. This can be associated with either lower inputs or output increases or vice versa. It is a positive sign for all hospitals in twenty regions of the Kingdom of Saudi Arabia that, faced with any new situation, they are creative in management and organizational policies, and will develop more in the coming days.

\section{ACKNOWLEDGMENT}

This project was supported by the Deanship of Scientific Research at Prince Sattam bin Abdulaziz University under the research project \# 2019/01/10007.

\section{REFERENCES}

[1] Mohamed Said Abou El-Seoud. Measuring Efficiency of Reformed Public Hospitals in Saudi Arabia: An Application of Data Envelopment Analysis, International Journal of Economics and Management Sciences,2013, Vol. 2, No. 9, 44-53

[2] M. Almalki, G. Fitzgerald and M. Clark. Health care system in Saudi Arabia: an overview, Eastern Mediterranean Health Journal, 2011, Vol. 17 No. 10,784-793 https://doi.org/10.26719/2011.17.10.784

[3] Mohamed Djerdjouri. Measuring Technical And Scale Efficiency Of Public Hospitals In The UAE Using The DEA Method: A Pilot Study, International Journal of Arts and Commerce, 2013, Vol. 2 No. 4, 1-10

[4] Asma M. A. Bahurmoz . Measuring Efficiency in Primary Health Care Centers in Saudi Arabia, J. KAU: Econ. \& Adm., 1998, Vol. 12, No. 2, 3-18

[5] Adel Mohammed Al-Shayea. Measuring hospital's units efficiency: A data envelopment analysis approach, International Journal of Engineering \& Technology IJETIJENS, 2011, Vol: 11 No: 06, 7-19

[6] Ibrahim Saeed Aqel and Khaled Atallah AlTarawneh. Study of The Relationship Between the Quality of the Medical Service and Patient Satisfaction in Private Hospitals Operating in Amman Area, European Journal of Business and Management, 2013, Vol.5, No.4, 255-74.

[7] Lee $\mathrm{K}$, Yang $\mathrm{S}$ and Choi $\mathrm{M}$. The association between hospital ownership and technical efficiency in a managed care environment, Journal of Medical Systems, 2009, 33:307-315.

[8] Banker, R.D., Charnes, A., Cooper, W.W. Some models for the estimation of technical and scale inefficiencies in Data Envelopment Analysis. Management Science, 1984, 30, 1078-1092. https://doi.org/10.1287/mnsc.30.9.1078

[9] Charnes, A., Cooper, W.W. and Rhodes, E.L. Measuring the Efficiency of Decision Making Units. European Journal of Operational Research, 1978, 2, 429-444.

[10]Hossein Moshiri, Syed Mohammed Al Junid, Rahmah Mohd Amin. Hospital Efficiency: Concept, Measurement Techniques and review of 
hospital efficiency studies, Malaysian Journal of Public Health Medicine, 2010, Vol. 10 (2): 35-43.

[11] María Caballer-Tarazona, Ismael Moya-Clemente, David Vivas-Consuelo, Isabel Barrachina-Martínez (2010). A model to measure the efficiency of hospital performance Mathematical and Computer Modelling, 2010, 52, 1095-1102.

[12] Tarig Hassan Mohammad Alamin, Atif Ali Yassin. Measuring Hospitals Efficiency using Data Envelopment Analysis Tool: Study on Governmental Hospitals Services at Ministry of Health - Khartoum State 2012, International Journal of Science and Research (IJSR), 2015, Volume 4 Issue 2, 1586-1592.

[13] Thomas N. Chirikos and Alan M. Sear. Measuring Hospital Efficiency: A Comparison of Two Approaches, HSR: Health Services Research, 2000, 34:6, 1389-1408.

[14] Alam Teg. Evaluating Efficiency of Hospitals in the Kingdom of Saudi Arabia: DEA-an Operational Research Technique Approach, International Journal of Mechanical Engineering and Technology, 2018, 9(10), pp. 1400- 1405.

[15] Muna Elsdaig, Dua' A. Nassar. Evaluation of Healthcare Information System Using Delone and McLean Quality Model, Case study KSA, International Journal of Advanced Trends in Computer Science and Engineering, 2019, Volume 8, No.1.4, pp.522-527. https://doi.org/10.30534/ijatcse/2019/8181.42019

[16] Intisar Shadeed Al-Mejibli, Nawaf Rasheed Alharbe. A Fuzzy Analytic Hierarchy Process for Security Risk Assessment of Web based Hospital Management System, International Journal of Advanced Trends in Computer Science and Engineering, 2019, Volume 8, No.5, pp.2470-2477. https://doi.org/10.30534/ijatcse/2019/92852019

[17] https://www.moh.gov.sa/en/Ministry/OpenData/Pag es/OpenDataLibrary.aspx 